\title{
Sistema de Emisión de Títulos Digitales: Módulo alumno y público en general
}

\section{Digital Degree Certificates Issuance System: Student and general public module}

\author{
VEGA-FLORES, Patricia†*, VEGA-OLVERA, Gustavo Ivan, ALCANTAR-ORTIZ, Patricia y \\ MORALES-OROZCO, David
}

Instituto Tecnológico Superior del Sur de Guanajuato. Av. Educación Superior No. 2000, Col. Benito Juárez, Uriangato, Gto.

ID $1^{\mathrm{er}}$ Autor: Patricia, Vega-Flores / ORC ID: 0000-0002-1375-5803

ID $1^{\text {er }}$ Coautor: Gustavo Ivan, Vega-Olvera / ORC ID: 0000-0002-0172-5383

ID $2^{\text {do }}$ Coautor: Patricia, Alcantar-Ortiz / ORC ID: 0000-0002-5667-3728

ID $3^{\text {er }}$ Coautor: David, Morales-Orozco / ORC ID: 0000-0002-3193-4709

DOI: $10.35429 / J U M .2019 .8 .3 .17 .24$

Recibido 10 de Marzo, 2019; Aceptado 30 de Junio, 2019

\begin{abstract}
Resumen
En los últimos años la Secretaría de Educación Pública (SEP) ha establecido nuevos estándares para la emisión de títulos profesionales electrónicos por las instituciones educativas y su registro ante la Dirección General de Profesiones (DGP). Actualmente, el Instituto Tecnológico Superior del Sur de Guanajuato (ITSUR) a través del cuerpo académico de Desarrollo de software bajo metodologías de ingeniería de software, se encuentra culminando la primera etapa de desarrollo de una aplicación para emitir, firmar y registrar títulos electrónicos ante la DGP. Esta aplicación no cuenta con ningún módulo que permita el acceso al alumno o público general interesado en la validez de los mismos. Por ello, se requiere desarrollar nuevos módulos que permitan al alumno dar seguimiento al proceso, descargar el título y la representación gráfica, así mismo permitir a empleadores o interesados comprobar la validez de un título. La metodología a usar será iterativa e incremental, para dividir el proyecto en iteraciones que generen incrementos de valor y lograr una estabilización en menor tiempo. Esta nueva versión tendrá mayor impacto, pues podrá ser distribuida a otros tecnológicos que han implementado el Sistema Integral Control Escolar (SICE) propiedad del ITSUR y así disminuir la brecha en la emisión de documentos electrónicos.
\end{abstract}

Software, Títulos Electrónicos, SICE

\begin{abstract}
In recent years, the Ministry of Public Education (SEP) has established new standards for the issuance of Digital Bachelor Degree Certificates titles by educational institutions and their registration with the Directorate General of Professions (DGP). Currently, the Technological Institute of the South of Guanajuato (ITSUR) through the academic body of Software Development under software engineering methodologies, is completing the first stage of developing an application to issue, sign and register digital bachelor degree certificates before the DGP. This application does not have any module that allows access to the student or general public interested in their validity. Therefore, it is required to develop new modules that require the student to follow up on the process, download the degree certificate and the graphic representation, as well as allow those employers or affected to verify the validity of a degree. The methodology to use will be iterative and incremental, to divide the project into iterations that generate increases in value and achieve stabilization in less time. This new version will have greater impact, as it may be distributed to other Technological Institutes that have implemented the Integral School Control System (SICE) owned by ITSUR and thus reduce the gap in the issuance of digital degree certificates.
\end{abstract}

Software, Digital Degree Certificate, SICE

Citación: VEGA-FLORES, Patricia, VEGA-OLVERA, Gustavo Ivan, ALCANTAR-ORTIZ, Patricia y MORALESOROZCO, David. Sistema de Emisión de Títulos Digitales: Módulo alumno y público en general. Revista de Gestión Universitaria. 2019. 3-8: 17-24

\footnotetext{
*Correspondencia del Autor (correo electrónico: p.vega@itsur.edu.mx)

$\dagger$ Investigador contribuyendo como primer autor.
} 


\section{Introducción}

Hoy en día, nos encontramos cada vez con mayor frecuencia ante cambios en las formas de realizar ciertos trámites. La mayoría de estos cambios implica que podamos realizarlos con mayor facilidad o rapidez, sobre todo porque muchos de ellos comienzan con una informatización de su proceso, proporcionando primeramente una agilización en el tiempo de atención, esto da paso a que posteriormente algunos de estos trámites permitan realizarse por el propio usuario a través de alguna aplicación por internet de forma que el usuario pueda realizarlo casi en cualquier momento y desde cualquier lugar. Así como menciona (El financiero, 2018) puedes solicitar la emisión dese una acta de nacimiento, cartas de antecedentes no penales, pagos de servicios, solicitudes de créditos, entre otros y todo sin necesidad de salir de tu casa.

Todo ello implica que las instituciones se preparen para poder proveer a los usuarios de plataformas que permitan realizar estos procedimientos, pero todas ellas deben asegurarse de que los requisitos que los usuarios deben reunir para poder realzar el trámite puedan también de cierta manera realizarse con mayor agilidad y sin trabas, de lo contrario el usuario terminará realizando el trámite de manera personal ocasionando que como menciona (Vanguardia, 2017) se afronten a "largas esperas, funcionarios que llevan trabajando muchas horas y que no siempre nos darán el trato que buscamos y el hecho de perder toda la mañana para algo muy sencillo".

Uno de los trámites que por indicación de la Secretaría de Educación Pública (SEP) deberá transformarse es la emisión de títulos profesionales y la cédula profesional, que ahora se manejarán en forma electrónica. Por ello todas las Instituciones de Educación Superior (IES) e Instituciones de Educación Media Superior (EMS), tanto públicas como privadas, deberán implementar ajustes en su proceso, de forma que permitan adecuarse a los nuevos estándares y procedimientos marcados por la SEP.

En el presente artículo se hablará de la implementación del Sistema de Emisión de Títulos Digitales, específicamente del módulo para el alumno y público en general, que se está realizando con el fin de alinearse a los estándares solicitados por la SEP.

\section{Antecedentes}

A partir del año 2018, el gobierno de la república derogó algunas disposiciones de la constitución política para dar inicio a la emisión de títulos profesionales electrónicos y cédulas electrónicas como se menciona en (Diario Oficial de la Federación, 2018) y así comenzar a brindar servicios más ágiles en cuanto al trámite de estos 2 documentos.

Esta tarea requiere mucha preparación, puesto que la SEP, tendría que trabajar en los mecanismos y estándares que permitieran a todas las IES e IEMS públicas y privadas, manejar un estándar uniforme (en lo posible) que permitiera hacer las validaciones correspondientes ante la Dirección General de profesiones (DGP).

Estos nuevos estándares para la emisión de títulos profesionales electrónicos por las instituciones educativas y su registro ante la DGP, han sido publicados en el Diario Oficial de la Federación (Diario Oficial de la Federación, 2018), y con la indicación de que las instituciones educativas deben dar inicio al proceso de transición de la emisión de títulos profesionales físicos a la emisión de títulos profesionales electrónicos, ya que una vez que el usuario tenga este último podrá realizar el trámite de la cédula profesional electrónica a través de un procedimiento completamente en línea.

El título profesional electrónico proveerá beneficios, principalmente para agilizar los trámites de cédula profesional electrónica, tal como indica el subsecretario de educación en (Tuirán, 2018):

- $\quad$ Se garantiza un servicio ágil y eficiente pues menciona que "el nuevo trámite se hace en cinco minutos a partir de que se acredita el pago de derechos".

- $\quad$ Es un trámite seguro y sin vulnerabilidad a la corrupción, es decir, ayudará a evitar la corrupción en el proceso de este tipo de trámites. además de que la cédula tiene elementos de seguridad, con mecanismos de fácil verificación.

- Los usuarios tendrán a su disposición una plataforma fácil de usar para sus trámites en la Dirección General de Profesiones. 
- También tendrán al alcance las innovaciones que las instituciones de educación superior implementen para el registro de títulos.

Eliminación de gastos de transporte o pagos de gestoría para los solicitantes de la cédula profesional.

El Tecnológico Nacional de México (TecNM) es una de las instituciones de educación superior más grandes de México y América Latina, cuenta con 262 instituciones en todo el país. De acuerdo al (SNETecNM, s.f.), el TecNM, tiene una cobertura de $13.11 \%$ de la matrícula de educación superior, alcanzando en nivel Licenciatura un $14.09 \%$ y en posgrado $1.41 \%$. En el gráfico 1, se muestra la distribución de la matrícula y la cobertura del TecNM a nivel licenciatura y postgrado, 262 de las 3704 IES del país son parte del TecNM, representando un $7.07 \%$ de las IES del país.

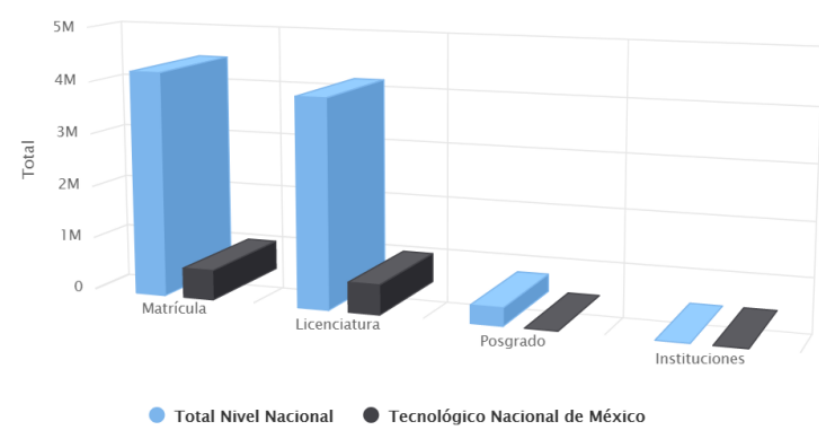

Gráfico 1 Participación del TecNM a nivel nacional Fuente: Obtenido de https://sne.tecnm.mx/public/nacional

El TecNM está trabajando en sus diversas instituciones para alinearse a los nuevos lineamientos de la SEP, algunos de los planteles precursores en este ámbito son:

- $\quad$ El Instituto Tecnológico de Chilpancingo que en Junio de 2018 expidió sus primeras cédulas profesionales a 14 egresados titulados.

El Instituto Tecnológico de Mérida en Marzo de 2019 generó los primeros títulos electrónicos del estado.

El Instituto Tecnológico de Querétaro en Marzo de 2019 emitió los primeros títulos electrónicos del estado.

Esta etapa de transición requiere de un trabajo arduo de todas las instituciones que paulatinamente se incorporarán al nuevo régimen establecido, pero al día de hoy aún falta la gran mayoría.
Por ejemplo, el estado de Guanajuato de acuerdo con el sitio de Siurp (Siurp SEP, s.f.), cuenta con 434 IES e IEMS, de las cuales el 13\% ya se encuentra en condiciones para emitir títulos electrónicos, pero solo el $5 \%$ ha emitido algún título electrónico, lo cual se puede observar en el gráfico 2 y la tabla 1 que se muestran abajo.

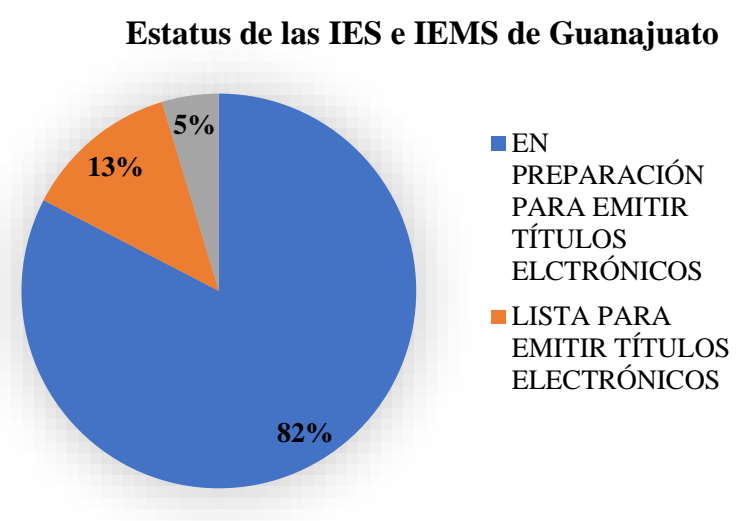

Gráfico 2 Situación actual de las IES e IEMS en Guanajuato ante la emisión de Títulos Electrónicos Fuente: Elaboración propia a partir de la información obtenida de https://siurp.sep.gob.mx/mvc/consultaInstituciones/irACo nsultaPorEntidad.htm

\begin{tabular}{|l|r|}
\hline \multicolumn{1}{|c|}{ Estatus } & Instituciones \\
\hline En preparación para emitir títulos electrónicos & $\mathbf{3 3 8}$ \\
\hline Lista para emitir títulos electrónicos & $\mathbf{5 2}$ \\
\hline Ya emite títulos electrónicos & $\mathbf{4 4}$ \\
\hline Total general & $\mathbf{4 3 4}$ \\
\hline
\end{tabular}

Tabla 1 Instituciones de Guanajuato ante la emisión de títulos electrónicos

Fuente: Elaboración propia a partir de la información obtenida de https://siurp.sep.gob.mx/mvc/consultaInstituciones/irACo nsultaPorEntidad.htm

El TecNM en el estado de Guanajuato cuenta con 10 instituciones, de las cuales el $70 \%$ ya están emitiendo títulos electrónicos y 3 aún se están preparando para ello, esto puede observarse en la Tabla 2.

Actualmente, el Instituto Tecnológico Superior del Sur de Guanajuato está supliendo esta necesidad a través de un servicio de terceros proporcionado por el Instituto Tecnológico de Celaya, pero al momento no ha podido realizar la emisión de títulos, ya que debe cumplir primeramente con ciertos prerrequisitos, principalmente para poder obtener las credenciales de autenticación para los servicios que ofrece la DGP. 
Situación de los Tecnológicos en Guanajuato

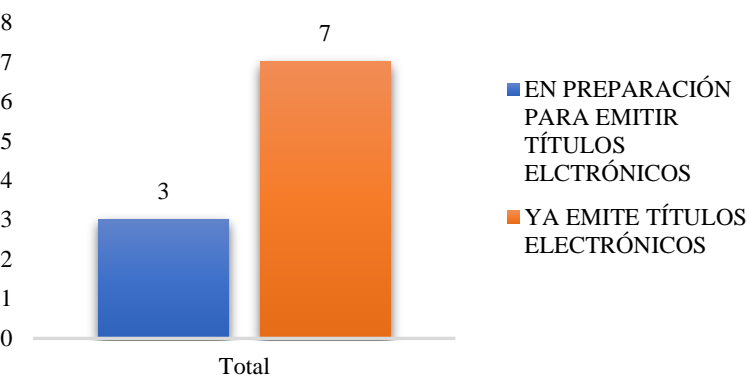

Tabla 2 Situación del TecNM en Guanajuato Fuente: Elaboración propia a partir de la información obtenida de https://siurp.sep.gob.mx/mvc/consultaInstituciones/irACo nsultaPorEntidad.htm

Actualmente, el Instituto Tecnológico Superior del Sur de Guanajuato (ITSUR) que forma parte del TecNM en Guanajuato, a través del cuerpo académico de Desarrollo de software bajo metodologías de ingeniería de software, se encuentra culminando la primera etapa de desarrollo de una aplicación para emitir, firmar y registrar títulos electrónicos ante la DGP, denominado como Sistema de Emisión de Títulos Digitales.

Esto debido a que el ITSUR cuenta con diversas aplicaciones que soportan diversos procesos del departamento de Servicios Escolares (Ver figura 1) y en lugar de depender de un tercero para realizar la emisión de títulos electrónicos ha preferido implementar un nuevo módulo que permita realizar esta tarea.

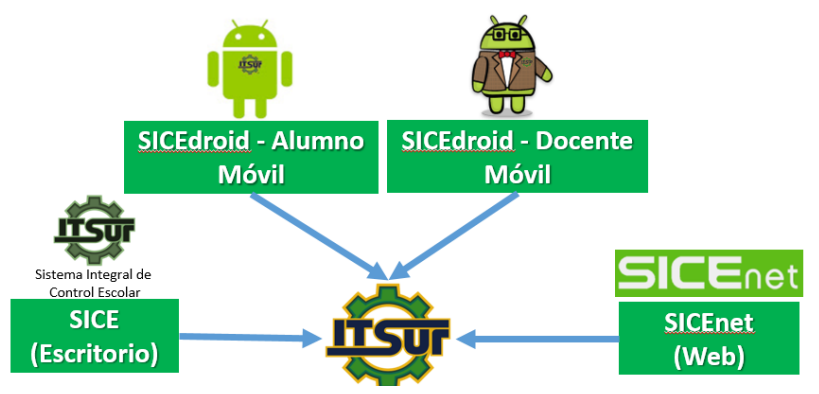

Figura 1 Ecosistema de aplicaciones de Servicios Escolares

Fuente: Elaboración Propia

En su primera versión, el Sistema de Emisión de Títulos Digitales no cuenta con ningún módulo que permita el acceso al alumno o público general interesado en la validez de los mismos y principalmente en verificar por ejemplo que el alumno se encuentra en proceso de titulación.
Por ello, se requiere desarrollar nuevos módulos que permitan al alumno dar seguimiento al proceso, descargar el título y su respectiva representación gráfica, así mismo permitir a empleadores o interesados comprobar la validez de un título o la comprobación del estatus de titulación del alumno. Estos módulos son importantes para brindar un mejor servicio al alumno una vez que ya inició su proceso de titulación.

\section{Metodología}

La metodología a usar será iterativa e incremental, para dividir el proyecto en iteraciones que generen incrementos de valor y lograr una estabilización en menor tiempo. Y que como menciona (Albaladejo, 2015), las entregas cortas y regulares permiten obtener una retroalimentación del cliente, lo que ocasionará que el producto desarrollado se vaya acercando a las expectativas del cliente. En la siguiente figura se muestra también, cómo debemos de manejar una comunicación constante con el cliente para poder ajustarse a las necesidades del cliente, situación que en el modelo tradicional es muy difícil de lograr.

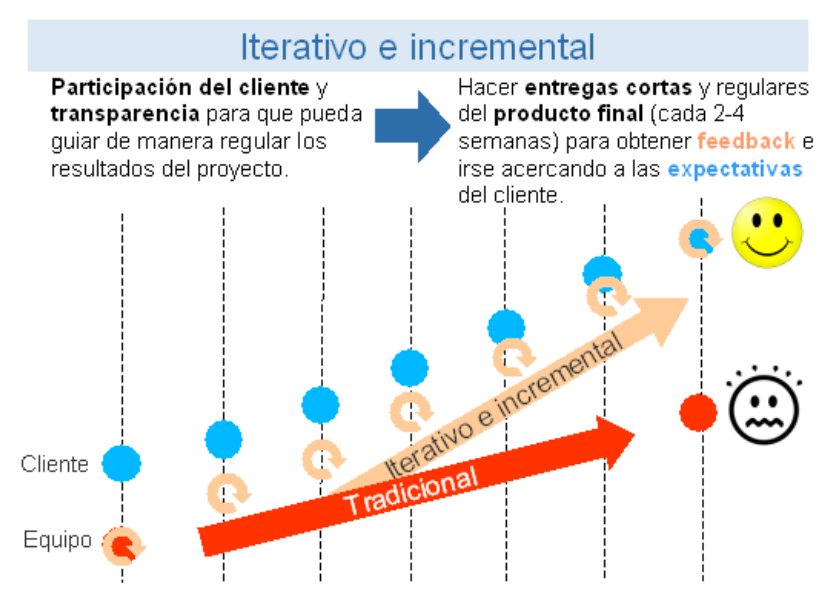

Figura 2 Integrando las expectativas del cliente con el trabajo de desarrollo

Fuente: Obtenida de https://proyectosagiles.org/iterativoincremental-control-empiricol

En el Sistema de Emisión de Títulos Digitales se incluye de manera general el registro de títulos electrónicos mediante lotes, pudiendo realizar los diversos pasos que requiere el proceso, tanto el firmado de los títulos, la generación del XML, el envío del lote a la DGP, consulta de estatus del lote y cancelación en la figura 2 se muestra una de las pantallas que permite generar los lotes de títulos en esta primera etapa. 


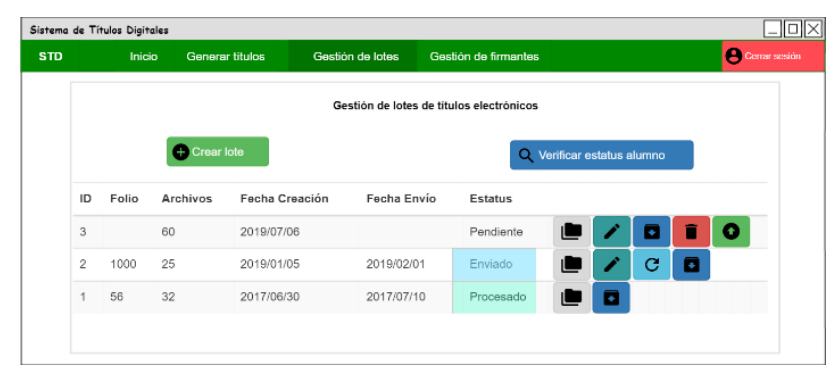

Figura 3 Generación de lotes de títulos de la 1ra etapa del Sistema de Emisión de Títulos Digitales

Fuente: Elaboración Propia

También se realizó un trabajo de homologación de catálogos y en general se incluyeron en el proyecto las funcionalidades mínimas requeridas para poderlo echar a andar en este año. Pero como ya se mencionó se incluyeron funcionalidades base, y se dejó fuera a uno de los principales interesados en este proceso: el alumno.

Por ello, se requiere la inclusión de módulos que permitan complementar el proceso, incluyendo a todos los interesados, principalmente el alumno.

Es requerido complementar el Sistema de Emisión de Títulos Digitales, incluyendo:

La revisión de estatus del proceso y la descarga del título tanto en XML como en formato gráfico, ya que, aunque no se requiera los alumnos consideran que el tener un título físico tiene un valor simbólico y emocional, además de que, en el proceso definido por la SEP que se muestra en la figura 3 , se considera que al profesionista deberá entregarse tanto en formato XML, como en formato gráfico (Dirección General de Profesiones).

Es importante que durante el proceso del trámite el alumno tenga una evidencia que permita demostrar la validez de los títulos hacia terceros.

Funcionalidades complementarias que faciliten la distribución de este módulo a otras instituciones que usan el Sistema SICE, módulos que permitan la gestión de información adaptable a cada institución.

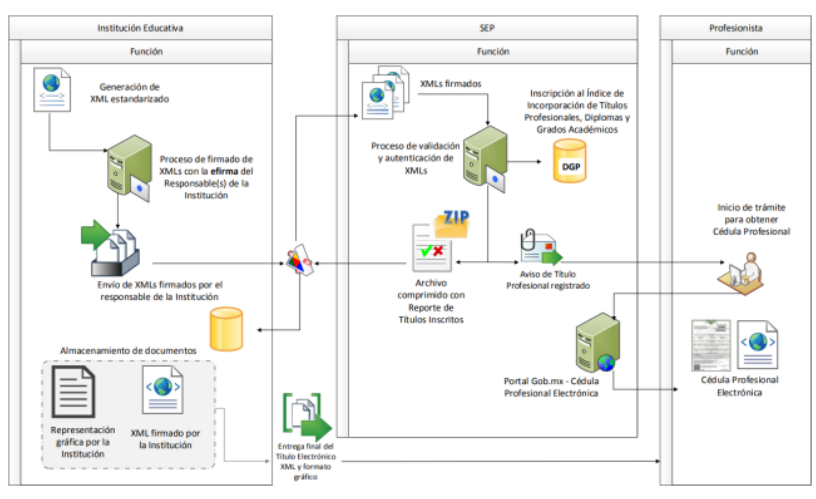

Figura 4 Proceso para la emisión de títulos electrónicos Fuente: Obtenido de https://www.sep.gob.mx/work/models/sep1/Resource/160 66/1/images/diagrama_proceso_general.pdf

Esta nueva versión tendrá mayor impacto, pues podrá ser distribuida a otros tecnológicos que han implementado el Sistema Integral Control Escolar (SICE) propiedad del ITSUR y así disminuir la brecha en la emisión de documentos electrónicos.

\section{Resultados}

Los requerimientos funcionales identificados para esta segunda etapa son los que se mencionan a continuación:

- $\quad$ Acceso del usuario alumno egresado con las mismas credenciales que cuando era alumno.

- Consulta del estado del trámite por parte del alumno, a fin de que pueda revisar por el mismo el avance en el proceso de trámite y validación de su título profesional electrónico.

- Edición del catálogo del alumno para incorporar foto de Título, ya que debido a que los títulos actuales son físicos, no se cuenta con una foto tipo titulo del alumno para la representación gráfica del mismo.

- Generación de la versión gráfica del Título, aunque el tpitulo se emitirá en su formato oficial en XML, es requerido contar con una representación gráfica imprimible que facilite la interpretación de los datos del título y que además siempre representará un valor añadido para el alumno.

- Descarga de título (XML y Gráfica) por parte del alumno, las veces que lo requiera.

Acceso de terceros a partir de un folio o url para verificar estado de titulación. 
Creación de módulos para gestionar los datos del tecnológico y permitir la homologación de datos, así como los datos de autenticación ante la Dirección General de Profesiones, con el fin de lograr una distribución de estos módulos a aquellos tecnológicos que cuentan con el SICE del ITSUR.

Estos requerimientos se han estado especificando mediante casos de uso, en la Tabla 3 se muestra un fragmento del caso de uso 9 referente la consulta del estatus del título.

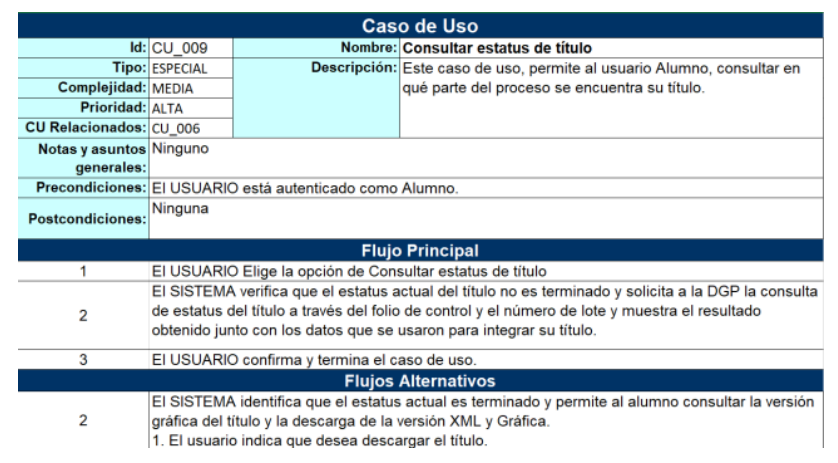

Tabla 3 Caso de uso 9, consultar estatus del título Fuente: Elaboración Propia

En cuanto a la arquitectura a manejar será la misma que para la primera etapa, puesto que es una adición a la funcionalidad, la aplicación se desarrollará bajo la plataforma Asp.NET con el uso de $\mathrm{C \#}$ como lenguaje del servidor y usando una arquitectura conformada por 3 capas, tal como se muestra en la figura 5 .

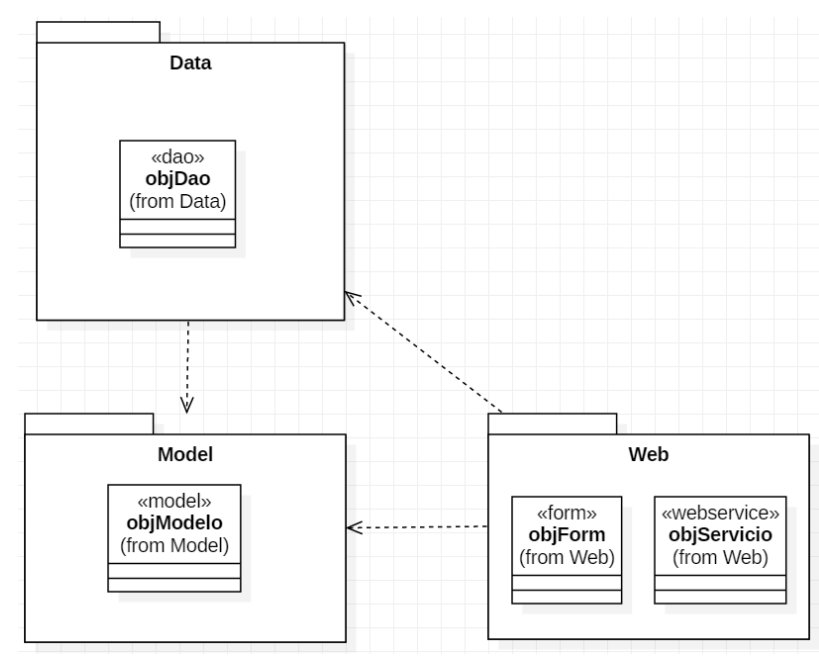

Figura 5 Arquitectura del Sistema de Emisión de Títulos Digitales

Fuente: Elaboración Propia

La capa DATA contendrá todos los componentes de tipo DAO (Data Access Object) que permitirán separar las operaciones de acceso a datos de la vista.
Esta capa se comunica con la base de datos mediante el uso de procedimientos almacenados. Esta técnica facilita mucho el mantenimiento de las aplicaciones, puesto que algunas adecuaciones solo requieren el cambio o ajuste de los procedimientos almacenados, sin la necesidad de manejar ningún despliegue de la aplicación web. La capa de MODEL solo contiene los modelos a partir de los cuales se intercambiará información entra las capas de DATA y WEB.

La capa de WEB, contendrá todos los elementos visuales y de programación del lado del cliente, además de los servicios web que en un futuro permitirán proveer a la aplicación móvil de SICEdroid Alumno de la posibilidad de obtener notificaciones sobre los títulos o consultar el estatus del título, lo que hace al ecosistema de aplicaciones más flexible.

Adicionalmente, esta aplicación funcionará con el patrón de Página Única, que como menciona en la página (Docs Microsoft, 2019) estas aplicaciones ejecutan la mayor parte de la lógica de la Interfaz Gráfica de Usuario (GUI) en un explorador web, aprovechando la potencia que tienen en la actualidad los equipos de cómputo. Para el desarrollo de cualquier aplicación (sobre todo al estar manejando incrementos) es imperativo usar algún gestor de versiones de manera que podamos ir estabilizando cada versión desplegada sin afectar el resto del desarrollo, por ello para este proyecto se tiene un repositorio del código del proyecto preparado con el gestor de versiones Git.

\section{Agradecimiento}

Al Tecnológico Nacional de México y a la Secretaría de Innovación, Ciencia y Educación Superior (SICES) por el apoyo al proyecto, mediante la asignación de recursos para el financiamiento del mismo.

\section{Conclusiones y trabajo future}

El desarrollo del proyecto mejorará el servicio proporcionado al alumno, facilitando tanto el trámite como el seguimiento del mismo. Además de que dotará al ITSUR de la posibilidad de alinearse a los nuevos estándares de emisión establecidos por la SEP y sobre todo poder generar sus propios Títulos Electrónicos desde una plataforma propietaria, que permita realizar ajustes sin la dependencia de un tercero.

VEGA-FLORES, Patricia, VEGA-OLVERA, Gustavo Ivan, ALCANTAR-ORTIZ, Patricia y MORALES-OROZCO, David. Sistema de Emisión de Títulos Digitales: Módulo alumno y público en general. Revista de Gestión Universitaria. 2019 
De igual forma, este proyecto ha impulsado el fortalecimiento del cuerpo académico Desarrollo de software bajo metodologías de ingeniería de software con clave ITESSGTO-CA-3, ya que este es el primer proyecto desarrollado con apoyo del TecNM. Sobre todo, generando productos de mayor impacto tanto dentro del ITSUR como fuera de él al implantar este sistema en otros tecnológicos.

El trabajo futuro será darle continuidad e implementar en este periodo el Sistema de Emisión de Títulos Digitales 1ra y 2da etapa, y en consecuencia distribuir este nuevo módulo en los Institutos Tecnológicos con el SICE (los cuales se muestran en la tabla 4), ya que por la nueva disposición todos se verán obligados a implementar un módulo similar, ayudando a disminuir la brecha en la emisión de documentos electrónicos.

\begin{tabular}{|l|l|}
\hline \multicolumn{2}{|c|}{ Institución Estado } \\
\hline $\begin{array}{l}\text { Instituto Tecnológico Superior de Ciudad } \\
\text { Hidalgo }\end{array}$ & Michoacán \\
\hline $\begin{array}{l}\text { Instituto Tecnológico Superior de } \\
\text { Tacámbaro }\end{array}$ & Michoacán \\
\hline Instituto Tecnológico Superior de Cocula & Jalisco \\
\hline Instituto Tecnológico Superior del Grullo & Jalisco \\
\hline Instituto Tecnológico Superior de Jerez & Zacatecas \\
\hline $\begin{array}{l}\text { Instituto Tecnológico Superior de } \\
\text { Tlatlahuquitepec }\end{array}$ & Puebla \\
\hline Escuela Normal de Chihuahua & Chihuahua \\
\hline
\end{tabular}

Tabla 4 Instituciones que cuentan con el SICE de ITSUR Fuente: Elaboración Propia

\section{Referencias}

Albaladejo, X. (24 de Septiembre de 2015). Eterativo-incremental Control empírico. Recuperado el 17 de Julio de 2019, de Proyectos ágiles: https://proyectosagiles.org/iterativoincremental-control-empirico/

Diario Oficial de la Federación. (13 de Abril de 2018). Aviso por el que se da a conocer el estándar para la recepción en forma electrónica de los títulos profesionales o grados académicos, para efectos de su registro ante la $D G P$. Obtenido de Diario Oficial de la Federación:

http://dof.gob.mx/nota_detalle.php?codigo $=551$ 9300\&fecha $=13 / 04 / 2018$
Diario Oficial de la Federación. (5 de Abril de 2018). DECRETO por el que se reforman y derogan diversas disposiciones del Reglamento de la Ley Reglamentaria del Artículo 50. Constitucional, relativo al ejercicio de las profesiones en el Distrito Federal. Obtenido de Diario Oficial de la Federación: http://www.dof.gob.mx/nota_detalle.php?codig $\mathrm{o}=5518146 \&$ fecha $=05 / 04 / 2018$

Dirección General de Profesiones. (s.f.). Diagrama del Proceso general para la emisión de Títulos Electrónicos. Recuperado el 10 de Agosto de 2019, de Secretaría de Educación Pública:

https://www.sep.gob.mx/work/models/sep1/Res ource/16066/1/images/diagrama_proceso_gener al.pdf

Docs Microsoft. (29 de Enero de 2019). Elección entre aplicaciones web tradicionales $y$ aplicaciones de página única (SPA). Recuperado el 20 de Agosto de 2019, de Docs Microsoft: https://docs.microsoft.com/eses/dotnet/architecture/modern-web-appsazure/choose-between-traditional-web-andsingle-page-apps

El financiero. (1 de Octubre de 2018). 5 trámites que puedes hacer por internet. Recuperado el 7 de Julio de 2019, de El financiero: https://www.elfinanciero.com.mx/nacional/5tramites-puedes-hacer-por-internet

Siurp SEP. (s.f.). Consulta de instituciones con títulos electrónicos registrados. Recuperado el 28 de Julio de 2019, de Siurp Sep: https://siurp.sep.gob.mx/mvc/consultaInstitucio nes/irAConsultaPorEntidad.htm

SNETecNM. (s.f.). Presencia del Tecnológico Nacional de México a nivel Nacional. Recuperado el 30 de Julio de 2019, de Sistema Nacional de Estadística TecNM: https://sne.tecnm.mx/public/nacional

Tecnológico Nacional de México. (s.f.). Historia. Recuperado el 1 de Agosto de 2019, de Tecnológico Nacional de México: https://www.tecnm.mx/historia.aspx 
Tuirán, R. (1 de Octubre de 2018). Comunicado 195.- Presenta SEP avances en cédula profesional electrónica, con seguridad, agilidad y transparencia; 1 de octubre. Recuperado el 10 de Julio de 2019, de Secretaría de Educación Pública:

https://www.gob.mx/sep/prensa/comunicado195-presenta-sep-avances-en-cedulaprofesional-electronica-con-seguridad-agilidad$\mathrm{y}$-transparencia-1-de-octubre?idiom $=e s$

Vanguardia. (23 de Enero de 2017). Algunos de los trámites que puedes hacer de manera online en el Gobierno de México. Recuperado el 7 de Julio de 2019, de Vanguardia: https://vanguardia.com.mx/articulo/algunos-delos-tramites-que-puedes-hacer-de-maneraonline-en-el-gobierno-de-mexico 